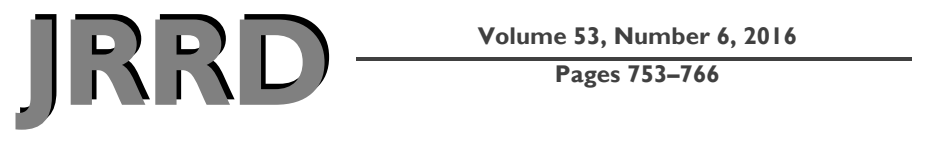

\title{
Functional performance differences between the Genium and C-Leg prosthetic knees and intact knees
}

\author{
CPT M. Jason Highsmith, PhD, DPT, CP(USAR); ${ }^{1-2^{*}}$ Jason T. Kahle, MSMS, CPO; ${ }^{1}$ Rebecca M. Miro, PhD; ${ }^{1}$ \\ M. Elaine Cress, PhD; ${ }^{3}$ Derek J. Lura, PhD; ${ }^{4}$ CDR William S. Quillen, PhD, DPT (USN Ret); ${ }^{1}$ Stephanie L. \\ Carey, PhD; ${ }^{5}$ Rajiv V. Dubey, PhD; ${ }^{5}$ Larry J. Mengelkoch, PhD, PT ${ }^{6}$ \\ ${ }^{1}$ School of Physical Therapy and Rehabilitation Sciences, University of South Florida, Tampa, FL; ${ }^{2}$ Department of \\ Veterans Affairs and Department of Defense Extremity Trauma \& Amputation Center of Excellence, Washington, DC; \\ ${ }^{3}$ Department of Kinesiology, University of Georgia, Athens, GA; ${ }^{4}$ Departments of Bioengineering and Software Engi- \\ neering, Florida Gulf Coast University, Fort Myers, FL; ${ }^{5}$ Mechanical Engineering Department, University of South \\ Florida, Tampa, FL; ${ }^{6}$ University of St. Augustine for Health Sciences, St. Augustine, FL
}

\begin{abstract}
Microprocessor prosthetic knees (MPKs) have advanced technologically, offering new features to decrease impairment and activity limitations for persons with transfemoral amputation (TFA). The Genium knee is functionally untested, and functional differences between it and intact knees are unknown. This study sought to determine whether Genium use improves functional performance compared with the C-Leg. A randomized experimental crossover design was used, with a cross-section of five nonamputee controls for comparison to normal. Twenty community-ambulating persons with TFA were trained and tested for accommodation with study components. All subjects $(n=25)$ were assessed using the Continuous-Scale Physical Functional Performance-10 (CS-PFP10) assessment. Subjects with TFA used both MPK systems. Genium use improved upper-body flexibility, balance, and endurance domain scores $(7.0 \%-8.4 \%, p<=0.05)$ compared with the CLeg. Only in the endurance domain did Genium users score significantly lower than nonamputees $(22.4 \%, p=0.05)$. Comparing the C-Leg with nonamputees, CS-PFP10 total (2.0\%-24.4\%, $p=$ 0.03 ) and all domains except upper-body strength were lower than nonamputees $(-13.4 \%$ to $-28.9 \%, p</=0.05)$. Nonetheless, regardless of knee condition, subjects with TFAs did not equal or surpass nonamputees in any functional domain, suggesting room for improvements in TFA functional performance.
\end{abstract}

Key words: activities of daily living, balance, endurance, flexibility, lower-limb amputation, microprocessor prosthetic knee, physical assessment, physical therapy, rehabilitation, strength.

\section{INTRODUCTION}

Studies of functional performance incorporating activities of daily living (ADLs) in persons with transfemoral amputation (TFA) are limited. Seymour et al. evaluated the performance of persons with TFA using two different prosthetic knees on an obstacle course simulating a home environment during loaded and unloaded conditions [1]. Subjects walked over varying surfaces (e.g., rug) and around objects (e.g., trash can) commonly found

\footnotetext{
Abbreviations: ADAPT $=$ Assessment of Daily Activities Performance in Transfemoral Amputees, ADL = activity of daily living, BAL = balance and coordination, CS-PFP10 = ContinuousScale Physical Functional Performance-10, END = endurance, ICC $=$ intraclass correlation coefficient, IRB $=$ institutional review board, LBS = lower-body strength, MPK = microprocessor prosthetic knee, $\mathrm{NMPK}=$ nonmicroprocessor knee, $\mathrm{SD}=$ standard deviation, TFA $=$ transfemoral amputation, UBF $=$ upper-body flexibility, UBS = upper-body strength.

*Address all correspondence to $\mathbf{M}$. Jason Highsmith, PhD, DPT, CP(USAR); University of South Florida, Morsani College of Medicine, School of Physical Therapy \& Rehabilitation Sciences, 12901 Bruce B. Downs Blvd, MDC 077, Tampa, FL 33612-4799; 813-974-3806; fax: 813-974-8915. Email: mhighsmi@health.usf.edu http://dx.doi.org/10.1682/JRRD.2014.06.0149
} 
within a home setting with hands free or while carrying a laundry basket. When subjects used a C-Leg (Ottobock; Duderstadt, Germany) microprocessor prosthetic knee (MPK), they completed the obstacle course more quickly and with fewer steps than when they used nonmicroprocessor knees (NMPKs). Similarly, Meier et al. designed a customized obstacle course involving many mobility skills including basic ambulation, stepping up, ramp ascent, turning, and walking across variably resistive surfaces [2]. C-Leg use tended to improve task completion time relative to a hydraulic swing-phase control polycentric knee. Task completion times were not so clearly improved relative to a single axis, fluid-controlled swing and stance knee system. Meier et al. found that use of the C-Leg decreased movement efficiency by 10 percent while completing the obstacle course in parallel with a mental loading task [2]. Course completion data regarding time and distance were very fractionalized. This likely increased group variance, clouding the ability to measure differences between conditions. Further, a curb step was used and referred to interchangeably as a "stair section" and "step section," which introduces some confusion and may not accurately reflect stair gait function. Nevertheless, Meier et al.'s data conflict with Seymour et al. and others, showing that C-Leg MPK usage increases walking speed during obstacle course performance, flat ground, and stairs [1-3]. Further, a recent systematic review's findings support improved gait efficiency with C-Leg MPK use in laboratory walking tests [4].

Given the uncertainty between obstacle course completion time relative to prosthetic-knee type, considering complex walking terrains were used in one study [2] and household floor surfaces in another [1], further assessment of household and functional tasks with MPK use is needed. Such assessments should more optimally test levels of activity and participation in accordance with the International Classification of Functioning, Disability and Health, as opposed to assessing at the body function and structure level. Singular task assessments (e.g., how fast a person walks over flat ground) test performance at the body function and structure level [5-6]. While individual task assessments provide insight into prosthetic optimization and success with specific singular tasks, assessment at higher activity levels and participation will provide greater insight into the integration of a prosthesis into a person's ADLs and their overall functional capabilities.

Theeven et al. designed the Assessment of Daily Activities Performance in Transfemoral Amputees
(ADAPT), a 17-item assessment scored by activity completion time and perceived difficulty [7]. The ADAPT's functional activities include simulated shopping, obstacle avoidance, stairs, and other community-based functional tasks. Theeven et al. report high test-retest reliability and sensitivity to change. Investigators evaluated performance on the ADAPT in persons with TFA who ambulated at fixed cadence [6]. Subjects used two MPKs (CLeg, Compact [Ottobock]) compared with their prestudy NMPK systems. When analyzed by entire sample, no difference in functional performance was found between knee conditions despite reporting the instrument's sensitivity to change. The investigators attributed this to high within-group variability. When stratified into functional subgroups (e.g., low, intermediate, and high function), high and intermediate functioning persons with TFA completed sections of the ADAPT significantly faster with C-Leg and Compact knee prostheses versus NMPKs. This was specific to the intermediate and high functioning subgroups relative to tasks requiring use of the upper limbs during movement [6].

In two [1,6] of the three [2] studies evaluating physical functional performance in simulated ADLs, the CLeg demonstrated some ability to outperform NMPKs. Limitations in these performance assessments include a lack of rigorous test validation and study-design issues necessitating nonparametric statistical analyses. Therefore, the contemporary literature unveils two specific dilemmas: first, whether MPKs truly make a favorable improvement in the functional performance of persons with TFA and second, which assessment best demonstrates this. To determine whether an MPK can improve functional performance of persons with TFA, novel MPK systems were considered.

The Genium MPK (Ottobock) has recently been introduced. The established C-Leg prosthetic knee system receives sensor input at $50 \mathrm{~Hz}$ and has an $8 \mathrm{MHz}$ microprocessor in addition to a knee angle sensor to determine sagittal knee position and an ankle moment sensor to determine sagittal moments about the ankle. Beyond these two sensors, four additional sensors are incorporated into the Genium system. The four additional sensors are an axial pylon load sensor, a sagittal knee moment sensor, a biaxial accelerometer, and a gyroscope. The cumulative functional effects of this sensor array are the subject of study here; however, some functions are proposed [8-9]. For instance, the accelerometer determines the direction of knee travel, and in concert with the gyroscope, the accelerometer assists in determining sagittal plane shank 
tilt and velocity to optimize knee resistance during hill walking. Genium's axial load sensor measures prosthetic weight bearing to facilitate knee movement resistance in specific functions. For instance, when the user is sitting, less resistance is desired to facilitate knee flexion. Oppositely, increased resistance to flexion may be desirable during prolonged weight bearing, such as standing to prepare a meal. Additionally, the Genium receives sensor input at an increased $100 \mathrm{~Hz}$ and has an $18.6 \mathrm{MHz}$ processing speed to facilitate increased responsiveness to changes in cadence, walking slope, posture, and movement cessation [8-9].

The Genium was preliminarily studied in a high functioning group of subjects [9]. Results from these preliminary studies show improvements at the body function and structure levels in persons with TFA following a very short $1 \mathrm{~d}$ accommodation with the Genium [5,8-9]. For instance, improvements were reported in stair ascent biomechanics, knee kinematics in gait at multiple speeds, and weight distribution in standing [8-9]. Initial subjective response from two separate studies have demonstrated improvements in family and social activities as well as in mobility and transportation with Genium use [10-11]. Specifically, stair and ramp ambulation as well as prolonged standing were perceived to be improved. While these results are promising, integrated functional performance capabilities at the activity and participation levels with the Genium are presently unknown. Given the Genium's objectively identified preliminary improvements over the C-Leg and its considerable design differences, the Genium was selected as the experimental prosthetic knee system in the current study to compare with the C-Leg, which is the most studied MPK.

To address the second dilemma of which functional assessment to use to test for differences between prosthetic knee systems, we selected the Continuous-Scale Physical Functional Performance-10 (CS-PFP10), in part for its face validity, in which participants perform tasks important in daily life in a serial fashion [12]. Although persons with TFA have not yet been assessed with the CS-PFP10, it has been used to assess multiple diagnostic groups including persons with Parkinson disease, heart failure, fibromyalgia, and wheelchair users, stroke survivors, and nondisabled elderly [12-18]. Further validation is evident by the instrument's domain scores being correlated $(p<0.05)$ with relevant measures of physical performance capacity [14]. For example, the lower-body strength (LBS) domain score is significantly correlated (intraclass correlation coefficient [ICC] $=0.69, p<0.05$ ) with knee extensor strength. A key difference in measuring physical performance domains in this way is that the test activities are familiar to patients in terms of their usual activities as opposed to isolated tests that have seemingly little relevance to patients. Additionally, the CS-PFP10 was capable of distinguishing between three functionally distinct groups of older persons: community dwellers and dependent and independent long-term care residents. The instrument's domain and total scores yielded high interrater (ICC $=0.92-0.99, p<0.01$ ) and test-retest (ICC $=0.85-0.97, p<0.01$ ) reliability as well [14]. The CS-PFP10 was determined to be sensitive to change, as evidenced by the ability to detect differences before and after a $12 \mathrm{wk}$ exercise program in a relatively small sample of nondisabled elderly ( $n=31$; delta index revealed moderate change [0.3-0.7]) [12]. High performance levels can be measured because no ceiling effects have been reported. The continuous scaling provides sensitivity to discriminate small differences with a small number of participants and allows the use of parametric statistical analysis [12]. The CS-PFP10 instrument is particularly useful for smaller-sample clinical therapeutic efficacy trials [12], making the test an ideal choice for this study.

Because the Genium knee incorporates additional sensors to determine axial load, slope, and direction of travel as well as knee moments and has demonstrated improvements at the body function and structure levels, we hypothesized that persons with TFA would experience improved ADL performance when using it compared with the C-Leg. We further hypothesized that control subjects would outperform persons with TFA in ADL function regardless of knee system used.

\section{METHODS}

The protocol was approved by the local institutional review board (IRB). Potential subjects were recruited by posting IRB-approved flyers including study information with local clinics, hospitals, and a national patient advocacy group. Investigators screened interested persons relative to eligibility criteria. For those with TFA, inclusion criteria were unilateral amputation through or above the knee, independent community ambulation including the ability to descend stairs and hills without support from a caregiver or assistive device, and $\geq 1$ yr of experience with 
a C-Leg MPK system. Those with TFA were excluded from participation if there was a history of skin breakdown, socket adjustments within $90 \mathrm{~d}$ of screening, or any comorbid cardiovascular or neurologic condition that would impair or preclude ambulation. Additionally, an inability to accommodate with the Genium knee by $90 \mathrm{~d}$ was an exclusion criterion. Control subjects were nonamputees and also had to be independent community ambulators with no comorbidities that would impair ambulatory function. Assistive device use was also an exclusion criterion among controls. All subjects had to be between 18 and $85 \mathrm{yr}$ of age in order to participate.

\section{Design Overview and Study Setting}

A randomized, experimental crossover design was used to compare functional differences between prosthetic knee conditions, and a cross-section of nonamputee controls was assessed for comparison to persons with TFA. The experiment was conducted in a university laboratory credentialed to conduct the CS-PFP10 assessment.

\section{Randomization and Interventions}

At enrollment, subjects were randomly assigned offsite to either continue with their C-Leg or be fitted with a Genium. On the day of knee assignment and fitting, the study prosthetist was notified of the assigned condition via telephone based on electronic random number generation (Microsoft Excel; Redmond, Washington).

Prosthetic fittings and adjustments were performed by a state-licensed prosthetist certified by the American Board for Certification in Orthotics, Prosthetics and Pedorthics as well as by Ottobock Healthcare for fitting both C-Leg and Genium MPK systems. Subjects' own prosthetic sockets and suspension systems remained constant throughout the experiment to prevent confounding from possible fit and acclimation issues. Once enrolled, subjects' usual prosthetic foot was exchanged for a Trias (Ottobock) or, in rare cases of height constraints, an Axtion foot (Ottobock) to control confounding. Subjects used the same foot throughout the experiment. Componentry alignment was set to manufacturer specifications and verified using the LASAR alignment system (Ottobock).

Following enrollment, anthropometric data were collected. Study foot and knee fittings and alignment recordings were conducted. All subjects then received a single initial training session with their respective initially randomized knee system and the study foot. During the initial training, subjects were assessed for the need for further training. Subjects were also invited to visit the study prosthetist and physical therapist as many times as necessary for further optimization of adjustments and alignment and additional training. Training included specific use in the functions of the study foot and both knee systems, in addition to transitional movements, obstacle crossing, as well as gait training on ramps, stairs, at variable speeds, and on variable surfaces. Training techniques used in this protocol are published elsewhere [1920]. Except for the uniformly administered initial session, additional training visits were counted and are reported in the results.

If subjects randomized to the C-Leg first, they were given 2 wk to receive their initial knee training and any necessary additional training as determined by the study therapist and prosthetist and to accommodate with the study foot prior to returning for phase A testing. Accommodation times for prosthetic feet are undefined and have ranged from $30 \mathrm{~min}$ [21] to $4 \mathrm{wk}$ [22]. Two weeks were deemed sufficient to accommodate with the study foot based upon two nonstudy subjects' successful foot accommodation during pilot testing in advance of the study. Subjects who randomized to the Genium first were tested between 2 wk and 3 mo following Genium fitting. Once fit with a Genium, subjects were contacted weekly to determine whether further training was necessary as well as their ability to walk without personal assistance on-

\section{Level ground.}

2. Inclines.

3. Declines.

4. Staircases (up and down).

5. Uneven ground.

Subjects were also invited to contact investigators at any point following the $2 \mathrm{wk}$ minimum to declare readiness to demonstrate accommodation as opposed to waiting for the scheduled, weekly telephone call. Once subjects verbally acknowledged the ability to ambulate independently on all five of the aforementioned terrains, they were scheduled to physically demonstrate the actual ability to ambulate on all terrains. This was the study accommodation test (adapted from Hafner et al. [23]). The duration from Genium fitting to assessment was tracked and is reported in the "Results" section. When subjects successfully performed the accommodation test, they were considered accommodated, and A-phase testing was scheduled. Following A-phase testing, the knees were switched, initial training was again provided for the second knee, and the process was repeated culminating in B-phase 
testing. As determined from preliminary testing, an inability to accommodate with the Genium knee within 3 mo was an exclusion criterion that would trigger removal from the study.

\section{Outcomes and Follow-Up}

The CS-PFP10 was administered adhering to the standardized operating procedure, including a standardized testing site, scripted dialog, and trained personnel. To mitigate rater bias, the study prosthetist, the investigator most knowledgeable in the study components, was not included as a rater. Complete CS-PFP10 instructions and laboratory layout measures are outlined and reported elsewhere [12]. CS-PFP10 scores 10 ADLs using time, distance, and mass. The raw data reflect the physiologic domains of function. The data collected per task and the domain to which they contribute are shown in Table 1. The test required approximately 30 min to complete. Raw data (time, distance, mass) are converted into summary scores using a validated algorithm within licensed scoring software. Scaled from 0 to 100 , the summary scores include the CS-PFP10 total score and five individual physiologic domain scores: upper-body strength (UBS), upper-body flexibility (UBF), balance and coordination (BAL), LBS, and endurance (END).

\section{Statistical Analysis}

All data were entered into a database and verified by the study statistician (blinded) prior to analysis. Shapiro-
Wilk test $(p>0.05)$ and a visual inspection of data histograms, normal Q-Q plots, and box plots were used to determine normality [25-26]. Descriptive statistics were calculated (e.g., means, standard deviations [SDs]) whenever possible. Because comparisons between MPK conditions were dependent, paired $t$-tests (two-tail) were used when data were normally distributed and at the interval or ratio scale level. If not, then the Wilcoxon Signed-Rank test for differences in medians was used. Because prosthetic knee groups and the control group were independent of each other, performance comparisons between these groups were made using independent samples $t$-tests with normally distributed data at the interval scale level or higher. Otherwise, the nonparametric equivalent, Wilcoxon signed-rank test for differences in medians was used. Effect sizes were calculated for group comparisons and interpreted as follows: 0.20 indicates a small effect size, 0.50 indicates a medium effect size, and 0.80 indicates a large effect size [27]. Statistical analyses were performed using SPSS version 22 (IBM Corporation; Armonk, New York), and the protocol's a priori level of significance was 0.05 .

\section{RESULTS}

\section{Subjects}

Twenty five consecutively screened subjects consented to participate and completed the study; 20 subjects

Table 1.

Tasks, functional domains, and metrics for the Continuous-Scale Physical Functional Performance-10 (CS-PFP10). The CS-PFP10 test scores each task by either mass, time, or distance.

\begin{tabular}{|c|c|c|c|c|c|c|}
\hline Task Difficulty & Task & UBS & LBS & UBF & BAL & END \\
\hline \multirow[t]{3}{*}{$\overline{\text { Low }}$} & 1. Pot carry $1 \mathrm{~m}$ & Mass & - & - & Time & - \\
\hline & 2. Don/doff jacket & - & - & Time & Time & - \\
\hline & 3. Vertical reach & - & - & Distance & - & - \\
\hline \multirow[t]{3}{*}{ Moderate } & 4. Pick up scarves from floor & - & Time & - & Time & - \\
\hline & 5. Floor sweep & - & Time & - & Time & - \\
\hline & $\begin{array}{l}\text { 6. Laundry: transfer clothes (a) washer to } \\
\text { dryer and (b) dryer to basket }\end{array}$ & Time & Time & - & Time & - \\
\hline & 8. Stair ascent/descent & - & Time & - & Time & - \\
\hline & 9. Carry groceries $70 \mathrm{~m}$ & Mass & Mass & 一 & Time & 一 \\
\hline & 10. 6 min walk test & - & - & - & - & Distance \\
\hline
\end{tabular}


had unilateral TFA, and 5 nonamputees served as controls. The five nonamputee controls included three males and two females with a mean \pm SD (range) age of $57.2 \pm$ $15.7 \mathrm{yr}(37-77 \mathrm{yr}$ ), body mass of $66.6 \pm 9.4 \mathrm{~kg}$ (54$78 \mathrm{~kg})$, and height of $170.2 \pm 8.6 \mathrm{~cm}(157-177 \mathrm{~cm})$. Two control subjects were retired, and three were employed. Controls were healthy, reporting no cardiovascular or musculoskeletal conditions.

The 20 subjects with TFA included 4 females and 16 males with a mean \pm SD (range) age of $46.5 \pm 14.2 \mathrm{yr}$ (24-75 yr), body mass of $82.0 \pm 15.9 \mathrm{~kg}(57-112 \mathrm{~kg})$, and height of $177.0 \pm 9.6 \mathrm{~cm}(154-192 \mathrm{~cm})$. Age and height were not significantly different between experimental and control subjects; however, body mass was significantly greater $(p=0.01)$ in subjects with TFA. The majority of subjects with TFA lost their leg because of trauma (15/20), four as a result of malignancy, and one due to peripheral vascular disease. Eleven of the subjects with TFA were employed, two were students, two were retired, and the remaining five were governmentally classified as being disabled. The mean time since amputation was $17.7 \pm 15.6$ yr (3-47 yr). The average residual limb length was $70 \pm 30$ percent (15\%-100\%) of the soundside femur, and the mean hip flexion contracture angle was $12.8^{\circ} \pm 7.7^{\circ}\left(0^{\circ}-27^{\circ}\right)$ as measured manually with a goniometer in the Thomas Test position [28]. Eighteen subjects used the Trias foot. The Axtion foot was used in two cases because of height constraints.

\section{Training and Accommodation}

All subjects successfully accommodated with both knee systems. Beyond the two initial functional training sessions (one per knee condition), subjects did not require or request further training or adjustments when using the C-Leg, evident by subjects demonstrating mas- tery of knee functions during initial training and by successfully completing the accommodation test. When using the Genium, subjects required $0.7 \pm 1.0$ additional visits (0-4) for postfitting prosthetic adjustments. Subjects additionally required $3.0 \pm 1.8$ visits (1-8) with the study physical therapist for functional training to master new features with the Genium knee, including stance locking (i.e., so-called intuitive stance), reciprocal stair ascent, obstacle crossing, and the stance flexion feature and required $67.9 \pm 27.1 \mathrm{~d}(18-119 \mathrm{~d})$ to successfully complete the accommodation test with the Genium knee system.

\section{Knee Alignment}

The mean distance between knee center and ground reaction force vector (sagittal knee alignment) when subjects used the C-Leg was $3.1 \pm 2.3 \mathrm{~cm}(-4.0$ to $8.0 \mathrm{~cm})$, where the force vector was anterior to knee center in the majority of cases. When using the Genium, sagittal knee alignment was $2.5 \pm 2.8 \mathrm{~cm}(-3.4$ to $6.8 \mathrm{~cm})$. Alignment data were normally distributed and not significantly different $(p>0.05)$ between knee conditions.

\section{Continuous-Scale Physical Functional Performance-10}

All 25 subjects completed the protocol, and no data were missing. The UBF score was significantly improved (7.0\%, $p=0.01$, effect size 0.45 ) when subjects used the Genium (71.5 \pm 10.3$)$ compared with the C-Leg (66.5 \pm 12.0) (Table 2, Figure). The balance and endurance scores were significantly improved as well. Genium use resulted in 7.6 percent ( $p=0.03$, effect size 0.28 ) and 8.4 percent ( $p=0.02$, effect size 0.32 ) improvements in these scores compared with use of the C-Leg. The CSPFP10 total score was also significantly improved $(p=$

Table 2.

Comparisons between control subjects and subjects with transfemoral amputation using two different microprocessor knee systems. Effect sizes are defined as small (0.20), medium (0.50), and large (0.80).

\begin{tabular}{|c|c|c|c|c|c|c|c|c|c|c|c|c|}
\hline \multirow[b]{2}{*}{ Comparison } & \multicolumn{2}{|c|}{ CS-PFP10 TOT } & \multicolumn{2}{|c|}{ UBS } & \multicolumn{2}{|c|}{ LBS } & \multicolumn{2}{|c|}{ UBF } & \multicolumn{2}{|l|}{ BAL } & \multicolumn{2}{|c|}{ END } \\
\hline & $\begin{array}{c}\% \\
\text { Difference }\end{array}$ & $\begin{array}{c}\text { Effect } \\
\text { Size }\end{array}$ & $\begin{array}{c}\% \\
\text { Difference }\end{array}$ & $\begin{array}{c}\text { Effect } \\
\text { Size }\end{array}$ & $\begin{array}{c}\% \\
\text { Difference }\end{array}$ & $\begin{array}{c}\text { Effect } \\
\text { Size }\end{array}$ & $\begin{array}{c}\% \\
\text { Difference }\end{array}$ & $\begin{array}{c}\text { Effect } \\
\text { Size }\end{array}$ & $\begin{array}{c}\% \\
\text { Difference }\end{array}$ & $\begin{array}{c}\text { Effect } \\
\text { Size }\end{array}$ & $\begin{array}{c}\% \\
\text { Difference }\end{array}$ & $\begin{array}{c}\text { Effect } \\
\text { Size }\end{array}$ \\
\hline Genium vs C-Leg & $7.4^{*}$ & 0.28 & 5.4 & 0.20 & 8.1 & 0.26 & $7.0^{*}$ & 0.45 & $7.6^{*}$ & 0.28 & $8.4^{*}$ & 0.32 \\
\hline Genium vs Control & 18.4 & 0.85 & 1.2 & 0.04 & 21.2 & 0.82 & 6.9 & 0.38 & 21.0 & 0.97 & $22.4^{*}$ & 1.05 \\
\hline C-Leg vs Control & $24.4^{*}$ & 1.17 & 6.5 & 0.25 & $27.6^{*}$ & 1.13 & $13.4^{*}$ & 0.71 & $27.1^{*}$ & 1.26 & $28.9^{*}$ & 1.39 \\
\hline
\end{tabular}

*Statistically significant differences $(p \leq 0.05)$.

BAL = balance and coordination, CS-PFP10 TOT = Continuous-Scale Physical Functional Performance-10 total score, END = endurance, LBS = lower-body strength, UBF = upper-body flexibility, UBS = upper-body strength. 


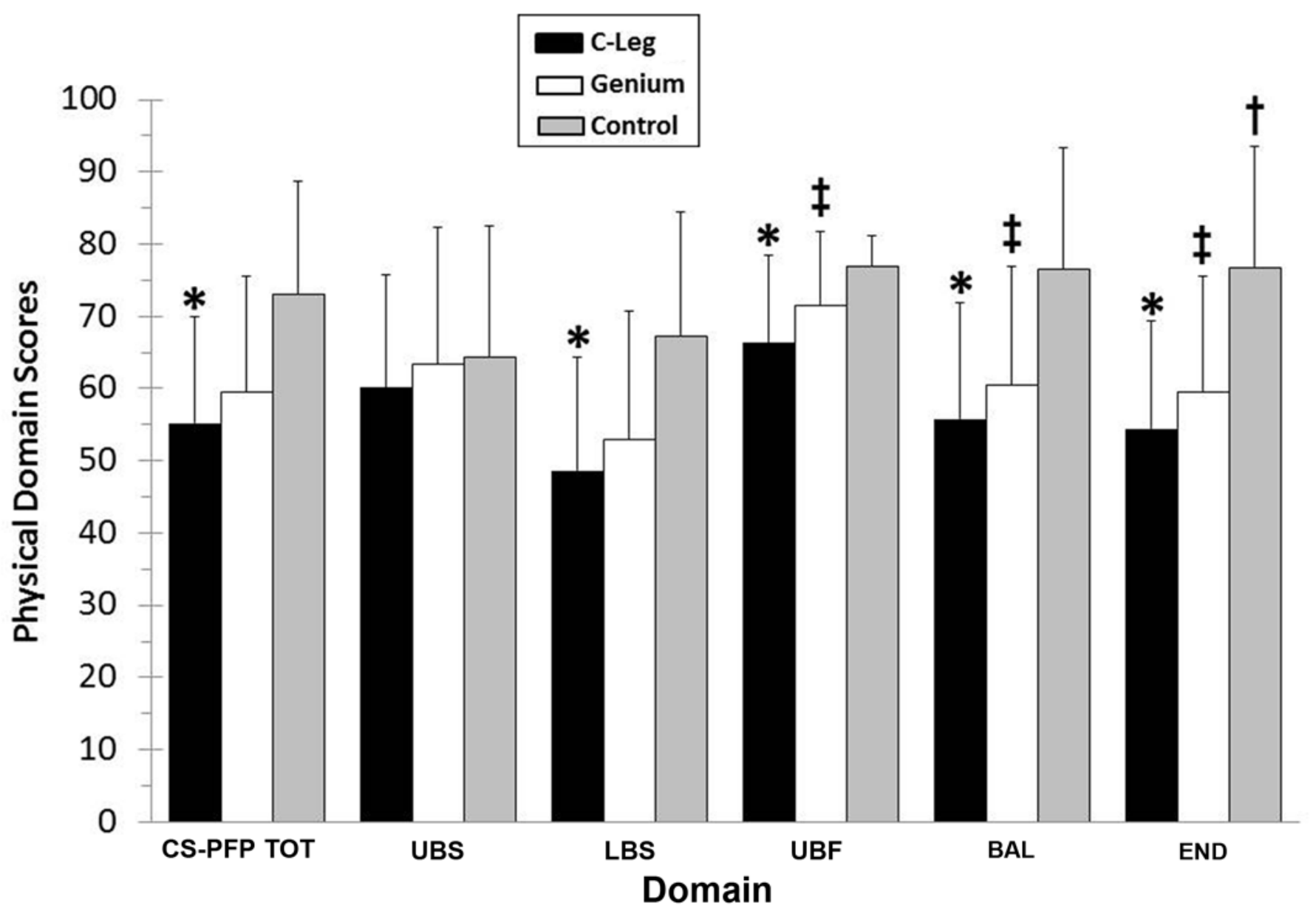

Figure.

Mean Continuous-Scale Physical Functional Performance-10 scores. Values are mean \pm standard deviation. * Statistically significant differences $(p \leq 0.05)$ between C-Leg knee condition and controls. ${ }^{\dagger}$ Statistically significant differences $(p \leq 0.05)$ between Genium knee condition and controls. ${ }^{\ddagger}$ Statistically significant differences $(p \leq 0.05)$ between Genium and C-Leg knee conditions. BAL $=$ balance and coordination, CS-PFP TOT = Continuous-Scale Physical Functional Performance total score, END = endurance, LBS $=$ lower-body strength, UBF = upper-body flexibility, UBS = upper-body strength.

0.03, effect size 0.28 ) by 7.4 percent with Genium use (59.6 \pm 16.0$)$ compared to C-Leg (55.2 \pm 14.8). While UBS and LBS scores did not attain the a priori level of significance, Genium use resulted in 5.4 percent ( $p=$ 0.09 ) and 8.1 percent ( $p=0.07$ ) improvements in these domains, respectively.

Nonamputee control subjects scored higher than subjects with TFA in all five domains and in CS-PFP10 total scores. No statistically significant difference was found between controls and the TFA group when using the Genium except in the endurance domain. Here, the difference was 22.4 percent $(p=0.05)$, with subjects with TFAs scoring $59.5 \pm 16.0$ and controls scoring $76.7 \pm$
16.9. The control group scored significantly higher than the TFA group in four of five domains, all except UBS when using the C-Leg. The smallest statistically significant difference in this comparison was in the UBF domain, which was 13.4 percent $(p=0.01)$. Control subjects scored $76.8 \pm 16.8$ when using the C-Leg, while subjects with TFA scored $66.5 \pm 12.0$. Conversely, the largest difference of statistical significance in comparing controls with the TFA group when using C-Leg was in the END domain, which was 28.9 percent $(p=0.01)$. The END domain score for controls was $76.7 \pm 16.9$ and $54.5 \pm$ 14.9 for TFA subjects using the C-Leg. 


\section{DISCUSSION}

The CS-PFP10 is established in terms of validity and reliability among older patients [14]. This study represents its first application in persons with TFA. Therefore, some level of face validation is justified in the absence of formal validation with this population. To begin with, we hypothesized a logical functional difference between nonamputee controls and subjects with TFA, and indeed this was the case. Specifically, with the Genium, the difference between subjects with TFA and controls ranged from 6.5 percent to 28.9 percent with C-Leg and 1.2 percent to 22.4 percent with Genium across all scores. The largest differences between control and subjects with TFA, regardless of MPK, were in the domains most heavily reliant upon lower-limb function: END, LBS, and BAL (Table 1). The domains with the smallest differences between controls and TFA were in the upper-body domains (UBS and UBF), as may be expected given lower-limb amputation. In other subject groups, the CSPFP10 has similarly demonstrated plausible scoring. For instance, progressively decreasing total scores in nondisabled controls stratified by age were identified, as were low scores in BAL and LBS in patients with Parkinson disease [13]. Face validity is evidenced in this study by the ability of the CS-PFP10 to detect difference in the lower-body domains, as one expects when comparing TFA with nondisabled controls. This increases confidence in the outcomes from the assessment.

All subjects in the study completed all CS-PFP10 tasks, suggesting no floor effect with this sample. The lowest (mean) domain score in this sample was 48.7 (LBS) from the TFA subjects when using the C-Leg. Further, none of the subjects reached peak scores on any activity or domain assessed. Specifically, the controls did not demonstrate a ceiling effect, providing additional evidence that differences identified in this study are real and not artificially constrained by limitations of the assessment. The highest domain score (mean) in the sample was 76.8 (UBF) from the nonamputee control group. Other studies similarly reported that the CS-PFP10 is at low risk of resulting in ceiling and floor effects. For instance, Manns et al. evaluated stroke survivors and reported no floor effects despite low scores ranging across CS-PFP10 domains from 12.8 (LBS) to 20.0 (UBS) [16]. The same study has so far reported the highest scores with this assessment in their control group (mean age $54 \mathrm{yr}$ ), with scores ranging across domains from 71.6 (LBS) to 79.3 (UBF), and no ceiling effects were reported. Unlike the UBS score in which no statistical differences between knees were observed, the UBF score significantly improved $(7.0 \%, p \leq 0.01)$ with the Genium. The effect size was at the upper end of the small to medium range for this comparison. Tasks contributing to UBF domain scoring include donning a jacket in the standing position and vertically reaching for maximal height to place an object on a shelf with both feet flat on the floor. In a sample of high-functioning household ambulators with TFA, Theeven et al. reported comparable improvements in upper-limb functional tasks in standing when using MPKs [6]. Tasks had functional similarity to those assessed here in terms of reaching up for shelving access to manipulate loads as well as grocery management. Further, tasks in their study were all performed in weight bearing and required successful balance, similar to this study. Authors discussed confidence as a major contributor to the improvements in upper-limb functionality [6]. Given that the Genium incorporates an axial load sensor to assist in altering knee stability by regulating movement resistance, it is possible that this feature contributed to improved upper-body reaching abilities when completed in weight bearing. Both UBF tasks-donning a jacket and vertically reaching-are completed while bilaterally weight bearing. In the Genium, weight bearing and lack of ambulation triggers increased knee stability by increasing flexion resistance. Thus, the user can load the prosthesis with assured confidence regarding knee stability, thus donning the jacket in less time and reaching higher within a wider base of support. Because of this stance locking feature (e.g., "intuitive stance”), the Genium can accept load during standing activities, whereas the C-Leg tends to slowly collapse into flexion under comparable loads. Simply stated, the more stable the base, the more upper-body activities may be supported. Because these tasks overlap fairly obviously with balance, they are demonstrative of the benefit of assessing integrated functional activities.

Findings in the LBS domain revealed an 8.1 percent improvement with the Genium that approached statistical significance $(p=0.07)$. Similar to UBS, contributions from actual torque production would be unlikely to change between the two knee systems since both are essentially passive. However, when measured in a functional context, other factors must be considered. Bellman et al. reported biomechanical improvements with Genium use in a study isolating stair gait [8]. Given that the LBS 
domain score is composed of six tasks including stair gait (Table 1) [12], it is possible that score improvements were influenced by stair gait biomechanics. Specific research regarding functional stair gait performance and stair gait during load carriage is necessary to corroborate Bellman et al.'s findings. Another study assessed stair ascent using the Ottobock X2 MPK compared with NMPKs [29]. The X2 and Genium are comparable regarding stair gait function, using flexion/extension resistance timing to maximize user climbing ability with their own strength as opposed to systems offering external-powered assistance [8,29-30]. Persons with TFA used a reciprocal stepping strategy with the X2, necessitating greater hip power, corroborating Bellman et al.'s findings $[8,29]$. While both studies demonstrate biomechanical improvements and control strategy with this system, neither has done so during functional task completion, particularly while carrying a load, an activity common in daily life. In this study, the LBS domain score also involved picking up four scarves from the floor from standing and getting up from the floor. Both of these tasks require considerable extensor force. The improvements in stair gait may have some functional carryover into these two tasks given that patients trained to utilize hip extensor force, which is permitted to enable knee extension. Additionally, floor sweeping was included, which many subjects completed while incorporating backward stepping. Backward stepping is another feature enabled by Genium that is not advised with the C-Leg system given the different toe loading requirements between these systems. Componentry and strength are both factors that can affect movement performance. For example, hip abductor strength training in persons with TFA improved functional performance in the Timed "Up and Go" test [31]. Both the Genium and the X2 system have demonstrated improvements in isolated stair ambulation, suggesting the component without strength training can change certain functional abilities, likely on a per-task basis. However, because LBS scores in this study were not significantly different between knees and the LBS domain tasks were multidimensional (e.g., stair walking with load carriage, rising from the floor), perhaps a combination of component, functional training, and strength training may be required to maximize integrated function and should be investigated.

There was a statistically significant 7.6 percent $(p=$ 0.03 ) improvement of small effect size (0.28) in BAL tasks with the Genium compared with C-Leg. These tasks include carrying loads, getting up from the floor, and changing positions, all in weight bearing. Compared with C-Leg, the Genium's sensor array including axial load data permits a more rapid transition between swing and stance mode [9]. This may enhance stability and improve confidence upon weight shifting because of locking or increasing flexion resistance under load to prevent a flexion collapse (e.g., fall). The improved functional outcome is an ability to complete tasks requiring quick postural changes and limb movements such as standing up from the floor in shorter time and with greater confidence [6]. Other tasks contributing to the BAL score include sweeping and changing laundry from washer to dryer. These tasks require multidirectional stepping, load carriage and manipulation, and small steps. Because the C-Leg requires a considerable toe load and knee extension moment to flex the knee for swing [9,32], subjects may have difficulty taking small steps and shifting load toward the prosthetic forefoot. This is due to difficulty meeting the switching criteria when stepping in confined spaces. In high functioning users, tasks requiring multiple small steps and forefoot loading (e.g., sweeping, changing laundry from washer to dryer) may be undertaken more cautiously with the C-Leg because of considerable switching failure instances resulting in low foot clearance [9]. In terms of CS-PFP10 performance, increased switching failure could lead to lower scores within the BAL and possibly other domains. In terms of functional implications, this could translate into impairments at the activity or possibly participation levels [5]. Specific multidirectional stepping studies including kinetic analyses are needed to learn more about toe loading practices in persons using the Genium. Improvements in the BAL domain represent multiple tasks that can improve a patient's functional level from independence with ADLs to independence with instrumental ADLs [33].

The END domain score is determined by the distance walked during a 6 min walk test, which has been used to validate numerous prosthetic functional assessments and provides a highly standardized endurance measure [34]. For the END domain, there was a statistically significant difference ( $p=0.02$, small effect size 0.32 ) of 8.4 percent with Genium use. Interestingly, short (e.g., $6 \mathrm{~m}$ ) and middistance (e.g., 38 and $75 \mathrm{~m}$ ) walking tests showed no difference in perceived exertion or time to completion between these knee systems [35]. The endurance requirement of repetitive walking for $6 \mathrm{~min}$ in addition to the duration of the entire CS-PFP10 test ( 30 min) is reflective of prolonged home and community ambulation. In 
this area, the Genium seems to provide an advantage over the C-Leg. This may enable an increase in activity participation through return to premorbid activities or exploration of new activities. One example might include sustained, aerobic exercise. One possible explanation for the increased END domain score is the improved swing phase kinematics identified by Bellman et al. [9]. They reported a consistent swing phase knee flexion angle regardless of walking speed. If a person with TFA has a sense of how the knee will flex and extend during swing phase when walking and the limb is not braking extension or flexion prematurely, perhaps the consistency permits a subtle but enhanced ability to sustain walking at a faster pace, as was found here. Ambulatory energy efficiency studies have not consistently reported improvements with the C-Leg compared with NMPKs [4]. Perhaps the Genium could improve energy efficiency or demand during prolonged walking. Further, studies have shown that walking exercise in persons with TFA will improve functional attributes such as aerobic capacity and gait speed [36-37]. Thus, walking exercise could improve the END domain and CS-PFP10 scores, but more importantly, functional level and participation in community and family activities.

Analyzing a sample of control subjects assisted in quantifying and visualizing the functional implications of TFA in terms of performing ADLs, and more specifically, which domains are most affected relative to nonamputees. The CS-PFP10 scores recorded for controls in this study (scores ranged 64.3-76.8) were reasonably similar to those of control subjects from other studies. For instance, Hearty et al. reported scores ranging from 58.7 to 65.0 in a control sample aged 55 to 64 yr [13]. Manns et al. reported a 71.6 to 79.3 score range in their control sample aged 54 yr (average) [16]. Differences between these samples can be accounted for by the differences in range of ages as well as sample sizes. Nevertheless, as may be expected, all domains except for UBS were significantly lower $(p<0.05)$ for persons with TFA using the C-Leg compared with controls. In contrast, when subjects with TFA accommodated with and used the Genium knee system, only the END domain was significantly lower ( $p=0.05)$ than the control group, thus reaffirming our initial hypothesis that Genium use would result in improved performance compared with the C-Leg. The effect size of having a TFA and using the C-Leg was medium to large in all domains (0.71-1.39) except for UBS (0.25) in comparison to the nonamputee control group. This reduced to small and medium effects with the Genium in the UBS (0.04) and UBF (0.38) domains, respectively. It is not surprising that the smallest differences (percentage and effect size) between TFA and controls were in domains involving the upper limbs. In terms of the CS-PFP10 total score, C-Leg use ultimately resulted in a significantly lower score compared with nonamputee controls $(p=0.03)$. However, use of the Genium system increased scores such that the difference between persons with TFA and nonamputee controls no longer reached statistical significance $(p=0.14)$, thus disproving our hypothesis from a statistical perspective. Essentially, a visually clear trend of functional improvement is identifiable between these two MPK systems, whereby the Genium system reduces functional and activity impairment in all domains tested in the direction of nonamputee controls (Figure). One final reference point further corroborates the functional difference between knee systems. Cress and Meyer identified an independence threshold of 57 (confidence interval: 47.858.6), whereby those scoring below 57 are at increased probability of dependence [38]. When using the C-Leg, subjects scored 55.2, compared with 59.6 with Genium use. Still, there is room for functional improvement in all categories. Perhaps further advancement of prosthetic technologies, associated rehabilitation, and surgical techniques will continue to close the functional gaps between those with TFA and nonamputees. It seems the domain least affected by TFA is UBS and the most impairment is identifiable within the END domain [37] regardless of knee system. Further, the Genium likely contributed to functional gains in the LBS, UBF, and BAL domains and thereby improved users' overall activity function, as evident in the overall CS-PFP10 score.

Subjects with TFA were trained to use the functions of both knee systems. Further, they were assessed for independence on a series of functional walking abilities in both knee systems prior to testing as evidence of their accommodation with both knee systems' respective and unique functions. Therefore, functional improvements as measured by CS-PFP10 scores were very likely attributable to true differences in performance and utilization of the knee systems as opposed to an order-of-effect bias or a learning effect simply from retesting. This is also strengthened by the randomized allocation. To objectively determine whether an order-of-effect or learning effect occurred, comparisons of mean individual domain and total CSPFP10 scores were compared post hoc for significant 
differences ( $p \leq 0.05$; paired, two-tailed $t$-tests) based on the sequence of testing as opposed to the knee used during the respective test. No statistically significant differences $(p>0.05)$ were observed in any domain or CS-PFP10 total scores based on the sequence of testing. This further rules out the risk of order-of effect and any learning effect. Moreover, alignments of the knee systems were set to manufacturer specification and similar between systems, thereby ruling out any stability advantages based purely on alignment of componentry.

\section{LIMITATIONS}

The CS-PFP10 has been used to assess function with at least six diagnostic groups; however, this study represents the first application in persons with TFA. While the assessment has proven to be valid, reliable, and sensitive to change in nonamputees, the specific psychometric properties, including minimal detectable change related to this unique group, are presently unknown and need to be established. Therefore, it is unclear whether the differences observed here meet the threshold for true change or are within the bounds of functional variance or measurement error. An additional consideration is the difference in duration for accommodation. Investigators attempted to mitigate this confounder by providing training and assessing for accommodation using industry-preferred methodology [39]. Beyond initial training provided for each knee, subjects were provided training when necessary and assessed for accommodation to assure optimal use of both knees. The result was a difference in the duration of accommodation and the number of training sessions beyond the initial training provided with each knee. To a large extent, these differences are unavoidable when subjects have previous experience with one technology and the comparator has different functions requiring mastery. Nonetheless, these differences are a factor worth considering when evaluating small performance differences between knee systems. Future studies could consider additional assessments or possibly a no-training group to formally assess training effects specifically. Also related to accommodation and training, the number of training sessions and duration of accommodation provided in this project were related to a group of independent community ambulators who had $\geq 1$ yr of experience with a C-Leg system. Therefore, the amount of training and duration of accommodation in persons functioning at different levels and transitioning to one of the study knee systems from a system not reported here may be completely different and should be considered with respect to the particular patient and their unique abilities. The study also did not incorporate blinding. Physical rehabilitation interventions are known to be particularly challenging to blind, but this should still be a goal in future studies [4041]. Additionally, only five nonamputee participants were studied, and their ages were diverse. A larger sample of controls would offer the ability to better match on key variables with the TFA group to better understand differences between the two groups.

\section{CONCLUSIONS}

There were no significant differences in functional UBS between nonamputees and persons with TFA regardless of knee condition. Compared with the C-Leg, Genium use improved the UBF, BAL, and END domains of functional performance, likely because of improved confidence, willingness to lift and carry greater mass, and ability to move faster during activity. These benefits may be technologically due to the incorporation of a faster processing speed and axial load data assisting in regulating knee resistance and offering new functions such as stance locking and backward stepping. In the LBS, UBF, BAL, and END domains, C-Leg use resulted in significantly lower scores compared with nonamputees. Genium use significantly reduced the magnitude of impairment. The only domain in which persons with TFA performed significantly lower than nonamputees regardless of knee condition was the END domain. In terms of total CS-PFP10 performance, C-Leg use resulted in significantly lower function compared with nonamputees, whereas Genium use was not significantly different from nonamputees. Nonetheless, regardless of knee condition, persons with TFA did not equal or surpass nonamputees in any functional performance domain, suggesting room for improvements in TFA integrated functional performance. Further, the CS-PFP10 test was able to detect statistically significant differences of small effect size between prosthetic knee conditions, which should be interpreted with caution because the test has not been formally assessed in persons with TFA. 


\section{ACKNOWLEDGMENTS}

\section{Author Contributions:}

Study concept and design: M. J. Highsmith, L. J. Mengelkoch. Acquisition of data: M. J. Highsmith, J. T. Kahle, R. M. Miro. Analysis and interpretation of data: M. J. Highsmith, D. J. Lura, S. L. Carey, L. J. Mengelkoch.

Drafting of manuscript: M. J. Highsmith, J. T. Kahle, R. M. Miro, L. J. Mengelkoch.

Critical revision of manuscript for important intellectual content: M. J. Highsmith, D. J. Lura, S. L. Carey, W. S. Quillen, R. V. Dubey, L. J. Mengelkoch.

Statistical analysis: M. J. Highsmith, L. J. Mengelkoch. Obtained funding: M. J. Highsmith.

Administrative, technical, or material support: M. J. Highsmith, R. M. Miro, M. E. Cress, L. J. Mengelkoch.

Study supervision: M. J. Highsmith, W. S. Quillen, R. V. Dubey.

Financial Disclosures: The sponsors had no role in the study design, manuscript writing, or decision to submit the manuscript for publication. The authors have declared that no competing interests exist.

Funding/Support: This material was based on work supported by the Florida High Tech Corridor/University of Southern Florida Connect (grant FHT 10-26) and Ottobock Healthcare (grant 6140101200).

Institutional Review: This study was approved by the University of South Florida IRB.

Participant Follow-Up: The authors do not plan to inform participants of the publication of this study. However, participants have been encouraged to check the study Web site for updated publications.

Disclaimer: Content of the manuscript represents the opinions of the authors and not necessarily those of the sponsors.

\section{REFERENCES}

1. Seymour R, Engbretson B, Kott K, Ordway N, Brooks G, Crannell J, Hickernell E, Wheeler K. Comparison between the C-leg microprocessor-controlled prosthetic knee and non-microprocessor control prosthetic knees: A preliminary study of energy expenditure, obstacle course performance, and quality of life survey. Prosthet Orthot Int. 2007; 31(1):51-61. [PMID:17365885] http://dx.doi.org/10.1080/03093640600982255

2. Meier MR, Hansen AH, Gard SA, McFadyen AK. Obstacle course: Users' maneuverability and movement efficiency when using Otto Bock C-Leg, Otto Bock 3R60, and CaTech SNS prosthetic knee joints. J Rehabil Res Dev. 2012; 49(4):583-96. [PMID:22773261] http://dx.doi.org/10.1682/JRRD.2010.05.0094

3. Kahle JT, Highsmith MJ, Hubbard SL. Comparison of nonmicroprocessor knee mechanism versus C-Leg on Prosthesis Evaluation Questionnaire, stumbles, falls, walking tests, stair descent, and knee preference. J Rehabil Res Dev. 2008;45(1):1-14. [PMID:18566922] http://dx.doi.org/10.1682/JRRD.2007.04.0054
4. Highsmith MJ, Kahle JT, Bongiorni DR, Sutton BS, Groer S, Kaufman KR. Safety, energy efficiency, and cost efficacy of the C-Leg for transfemoral amputees: A review of the literature. Prosthet Orthot Int. 2010;34(4):362-77. [PMID:20969495]

http://dx.doi.org/10.3109/03093646.2010.520054

5. Kohler F, Cieza A, Stucki G, Geertzen J, Burger H, Dillon MP, Schiappacasse C, Esquenazi A, Kistenberg RS, Kostanjsek N. Developing Core Sets for persons following amputation based on the International Classification of Functioning, Disability and Health as a way to specify functioning. Prosthet Orthot Int. 2009;33(2):117-29. [PMID:19367515] http://dx.doi.org/10.1080/03093640802652029

6. Theeven P, Hemmen B, Rings F, Meys G, Brink P, Smeets $\mathrm{R}$, Seelen H. Functional added value of microprocessorcontrolled knee joints in daily life performance of Medicare Functional Classification Level-2 amputees. J Rehabil Med. 2011;43(10):906-15. [PMID:21947182]

http://dx.doi.org/10.2340/16501977-0861

7. Theeven P, Hemmen B, Stevens C, Ilmer E, Brink P, Seelen $\mathrm{H}$. Feasibility of a new concept for measuring actual functional performance in daily life of transfemoral amputees. J Rehabil Med. 2010;42(8):744-51. [PMID:20809056] http://dx.doi.org/10.2340/16501977-0591

8. Bellmann M, Schmalz T, Ludwigs E, Blumentritt S. Stair ascent with an innovative microprocessor-controlled exoprosthetic knee joint. Biomed Tech (Berl). 2012;57(6): 435-44. [PMID:23241569] http://dx.doi.org/10.1515/bmt-2011-0029

9. Bellmann M, Schmalz T, Ludwigs E, Blumentritt S. Immediate effects of a new microprocessor-controlled prosthetic knee joint: A comparative biomechanical evaluation. Arch Phys Med Rehabil. 2012;93(3):541-49. [PMID:22373937] http://dx.doi.org/10.1016/j.apmr.2011.10.017

10. Highsmith MJ, Kahle JT, Miro RM, Lura DJ, Dubey RV, Carey SL, Quillen WS, Mengelkock LJ. Perceived differences between the Genium and the C-Leg microprocessor prosthetic knees in prosthetic-related function and quality of life. Technol Innov. 2014;15(4):369-75.

http://dx.doi.org/10.3727/194982413X13844489091297

11. Kannenberg A, Zacharias B, Mileusnic M, Seyr M. Activities of daily living: Genium bionic prosthetic knee compared with C-Leg. J Prosthet Orthot. 2013;25(3):110-17. http://dx.doi.org/10.1097/JPO.0b013e31829c221f

12. Cress ME, Petrella JK, Moore TL, Schenkman ML. Continuous-scale physical functional performance test: Validity, reliability, and sensitivity of data for the short version. Phys Ther. 2005;85(4):323-35. [PMID:15794703]

13. Hearty TM, Schenkman ML, Kohrt WM, Cress ME. Continuous scale physical functional performance test: Appropriateness for middle-aged adults with and without 
Parkinson’s disease. J Neurol Phys Ther. 2007;31(2):64-70. [PMID:17558359]

http://dx.doi.org/10.1097/NPT.0b013e3180676afa

14. Cress ME, Buchner DM, Questad KA, Esselman PC, deLateur BJ, Schwartz RS. Continuous-scale physical functional performance in healthy older adults: A validation study. Arch Phys Med Rehabil. 1996;77(12):1243-50.

[PMID:8976306] http://dx.doi.org/10.1016/S0003-9993(96)90187-2

15. Cress ME, Kinne S, Patrick DL, Maher E. Physical functional performance in persons using a manual wheelchair. J Orthop Sports Phys Ther. 2002;32(3):104-13.

[PMID:12168737]

http://dx.doi.org/10.2519/jospt.2002.32.3.104

16. Manns PJ, Tomczak CR, Jelani A, Cress ME, Haennel R. Use of the continuous scale physical functional performance test in stroke survivors. Arch Phys Med Rehabil. 2009;90(3):488-93. [PMID:19254616] http://dx.doi.org/10.1016/j.apmr.2008.08.219

17. Panton LB, Kingsley JD, Toole T, Cress ME, Abboud G, Sirithienthad P, Mathis R, McMillan V. A comparison of physical functional performance and strength in women with fibromyalgia, age- and weight-matched controls, and older women who are healthy. Phys Ther. 2006;86(11): 1479-88. [PMID:17079747]

http://dx.doi.org/10.2522/ptj.20050320

18. Gary RA, Cress ME, Higgins MK, Smith AL, Dunbar SB. A combined aerobic and resistance exercise program improves physical functional performance in patients with heart failure: A pilot study. J Cardiovasc Nurs. 2012; 27(5):418-30. [PMID:21912268] http://dx.doi.org/10.1097/JCN.0b013e31822ad3c3

19. Highsmith MJ, Kahle JT, Lewandowski AL, Kim SH, Mengelkoch LJ. A method for training step-over-step stair descent gait with stance yielding prosthetic knees. J Prosthet Orthot. 2012;24(1):10-15. http://dx.doi.org/10.1097/JPO.0b013e3182436628

20. Highsmith MJ, Kahle JT, Lura DJ, Lewandowski AL, Quillen WS, Kim SH. Stair ascent and ramp gait training with the Genium knee. Technol Innov. 2014;15(4):349-58. http://dx.doi.org/10.3727/194982413X13844488879267

21. Underwood HA, Tokuno CD, Eng JJ. A comparison of two prosthetic feet on the multi-joint and multi-plane kinetic gait compensations in individuals with a unilateral transtibial amputation. Clin Biomech (Bristol, Avon). 2004; 19(6):609-16. [PMID:15234485]

http://dx.doi.org/10.1016/j.clinbiomech.2004.02.005

22. Perry J, Boyd LA, Rao SS, Mulroy SJ. Prosthetic weight acceptance mechanics in transtibial amputees wearing the Single Axis, Seattle Lite, and Flex Foot. IEEE Trans Rehabil Eng. 1997;5(4):283-89. [PMID:9422453] http://dx.doi.org/10.1109/86.650279
23. Hafner BJ, Willingham LL, Buell NC, Allyn KJ, Smith DG. Evaluation of function, performance, and preference as transfemoral amputees transition from mechanical to microprocessor control of the prosthetic knee. Arch Phys Med Rehabil. 2007;88(2):207-17. [PMID:17270519] http://dx.doi.org/10.1016/j.apmr.2006.10.030

24. Cress ME, Buchner DM, Questad KA, Esselman PC, deLateur BJ, Schwartz RS. Exercise: Effects on physical functional performance in independent older adults. J Gerontol A Biol Sci Med Sci. 1999;54(5):M242-48. [PMID:10362007] http://dx.doi.org/10.1093/gerona/54.5.M242

25. Razali NM, Wah YB. Power comparisons of Shapiro-Wilk, Kolmogorov-Smirnov, Lilliefors and Anderson-Darling tests. J Statistical Modeling Analytics. 2011;2(1):21-33.

26. Shapiro SS, Wilk MB. An analysis of variance test for normality (complete samples). Biometricka. 1965;52(3/4): 591-611. http://dx.doi.org/10.1093/biomet/52.3-4.591

27. Cohen J, editor. Statistical power analysis for the behavioral sciences. 2nd ed. Hillsdale (NJ): L. Erlbaum Associates; 1988.

28. Magee DJ. Orthopedic physical assessment. 4th ed. St. Louis (MO): Saunders Elsevier; 2006.

29. Aldridge Whitehead JM, Wolf EJ, Scoville CR, Wilken JM. Does a microprocessor-controlled prosthetic knee affect stair ascent strategies in persons with transfemoral amputation? Clin Orthop Relat Res. 2014;472(10):3093-3101.

[PMID:24515402] http://dx.doi.org/10.1007/s11999-014-3484-2

30. Highsmith MJ, Kahle JT, Carey SL, Lura DJ, Dubey RV, Csavina KR, Quillen WS. Kinetic asymmetry in transfemoral amputees while performing sit to stand and stand to sit movements. Gait Posture. 2011;34(1):86-91.

[PMID:21524913]

http://dx.doi.org/10.1016/j.gaitpost.2011.03.018

31. Pauley T, Devlin M, Madan-Sharma P. A single-blind, crossover trial of hip abductor strength training to improve Timed Up \& Go performance in patients with unilateral, transfemoral amputation. J Rehabil Med. 2014;46(3):264-70.

[PMID:24363039]

http://dx.doi.org/10.2340/16501977-1270

32. Segal AD, Orendurff MS, Klute GK, McDowell ML, Pecoraro JA, Shofer J, Czerniecki JM. Kinematic and kinetic comparisons of transfemoral amputee gait using C-Leg and Mauch SNS prosthetic knees. J Rehabil Res Dev. 2006; 43(7):857-70. [PMID:17436172] http://dx.doi.org/10.1682/JRRD.2005.09.0147

33. American Physical Therapy Association. Guide to physical therapist practice. 2nd ed. Alexandria (VA): American Physical Therapy Association; 2001.

34. Kark L, McIntosh AS, Simmons A. The use of the 6-min walk test as a proxy for the assessment of energy expenditure during gait in individuals with lower-limb amputation. 
JRRD, Volume 53, Number 6, 2016

Int J Rehabil Res. 2011;34(3):227-34. [PMID:21654324] http://dx.doi.org/10.1097/MRR.0b013e328346e893

35. Highsmith MJ, Kahle JT, Lura DJ, Dubey RV, Carey SL, Quillen WS, Mengelkoch LJ. Short and mid-distance walking and posturography with a novel microprocessor knee. Technol Innov. 2014;15(4):359-68. http://dx.doi.org/10.3727/194982413X13844488879302

36. Darter BJ, Nielsen DH, Yack HJ, Janz KF. Home-based treadmill training to improve gait performance in persons with a chronic transfemoral amputation. Arch Phys Med Rehabil. 2013;94(12):2440-47. [PMID:23954560] http://dx.doi.org/10.1016/j.apmr.2013.08.001

37. Wezenberg D, van der Woude LH, Faber WX, de Haan A, Houdijk H. Relation between aerobic capacity and walking ability in older adults with a lower-limb amputation. Arch Phys Med Rehabil. 2013;94(9):1714-20.

[PMID:23466292] http://dx.doi.org/10.1016/j.apmr.2013.02.016

38. Cress ME, Meyer M. Maximal voluntary and functional performance levels needed for independence in adults aged 65 to 97 years. Phys Ther. 2003;83(1):37-48. [PMID:12495411]

39. Highsmith MJ. Microprocessor knees: Considerations for accommodation and training. J Prosthet Orthot. 2013;25(4S): 60-64.

http://dx.doi.org/10.1097/JPO.0b013e3182a891b5
40. Johnston MV, Sherer M, Whyte J. Applying evidence standards to rehabilitation research. Am J Phys Med Rehabil. 2006;85(4):292-309. [PMID:16554683]

http://dx.doi.org/10.1097/01.phm.0000202079.58567.3b

41. Whyte J. Clinical trials in rehabilitation: What are the obstacles? Am J Phys Med Rehabil. 2003;82(10 Suppl): S16-21. [PMID:14502034] http://dx.doi.org/10.1097/01.PHM.0000086992.95121.C2

Submitted June 17, 2014. Accepted in revised form November 13, 2015.

This article and any supplementary material should be cited as follows:

Highsmith MJ, Kahle JT, Miro RM, Cress ME, Lura DJ, Quillen WS, Carey SL, Dubey RV, Mengelkoch LJ. Functional performance differences between the Genium and C-Leg prosthetic knees and intact knees. J Rehabil Res Dev. 2016;53(6):753-66.

http://dx.doi.org/10.1682/JRRD.2014.06.0149

ResearcherID: Derek J. Lura, PhD: E-8210-2011

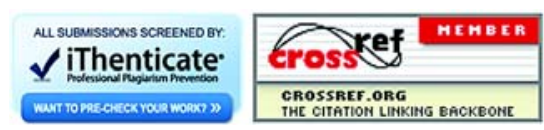

